\title{
BOUNDED INFLUENCE REGRESSION IN THE PRESENCE OF HETEROSKEDASTICITY OF UNKNOWN FORM
}

\author{
Miguel A. DELGADO \\ Department of Economics \\ Indiana University \\ Bloomington, IN 47405 USA
}

\begin{abstract}
In a regression model with conditional heteroskedasticity of unknown form, we propose a general class of M-estimators scaled by nonparametric estimates of the conditional standard deviations of the dependent variable. We give regularity conditions under which these estimators are asymptotically equivalent to M-estimators scaled by the true conditional standard deviations. The practical performance of these estimators is investigated through a Monte Carlo experiment.
\end{abstract}

\section{Introduction}

Cross-sectional data are typically heteroskedastic, of ten containing aberrant observations or gross errors. Under these circumstances, the least squares estimation method produces inefficient estimates. It is difficult to determine the source of inefficiency. As Huber (1973) pointed out:

$\because \quad$ 'In the regression case, uncontrollable inhomogeneity of

variance among the disturbances and genuinely long tailed error

distributions have almost indistinguishable effects both

impairing the efficiency of the estimates'.

Hence, it seems worthwhile to correct for heteroskedasticity using robust regression analysis. Carroll and Ruppert (1982) considered M-estimators scaled differently for each observation. The scale estimators were obtained under prior information on the functional form of the heteroskedasticity. Simulations reported by Carroll and Ruppert (1982) are encouraging. These estimators are expected to be nonrobust when the parameterization of the heteroskedasticity is incorrect. Asymptotically efficient estimators in the presence of unknown heteroskedasticity have been obtained by Carroll (1982), Robinson (1987) and Delgado (1989). However, these estimators have an unbounded influence function to residuals and leverage.

In this paper we establish the asymptotic properties of M-estimators with bounded influence function in the presence of heteroskedasticity of unknown nature. The conditional scale estimates are consistent nonparametric estimates of the conditional standard deviation of the dependent variable. The rest of the paper is organized as follows. In the next section we discuss a general class of optimal M-estimators which scale by the true conditional standard deviation of the dependent variable. In section 3 we present our M-estimators. 
In section 4 we give conditions under which these M-estimators are adaptive. In section 5 we report the results of a Monte Carlo experiment and in section 6 we sumarize our conclusions. Proofs are confined to the appendix.

\section{Optimal Bounded Regression in the Presence of Heteroskedasticity}

We consider the usual regression model. We have independent observations $\left\{\left(\mathrm{Y}_{1}, \mathrm{X}_{1}\right), 1 \leq \mathrm{i}\right.$ ) $\left.\mathrm{n}\right\}$ from $a \mathbb{R} \times{ }^{\mathrm{P}_{\mathbb{R}}}$ random variable $(\mathrm{Y}, \mathrm{X})$ where,

$$
Y_{i}=X_{i}^{\prime} \theta^{0}+\varepsilon_{i} \sigma\left(X_{i}\right) ; i \geq 1
$$

and $\theta^{\circ}$ is a pxl vector of unknown parameters, $\sigma($.$) is an unknown function and$ $\varepsilon 1$ are, conditionally on $\mathrm{X}_{1}$, symmetric about zero with $\operatorname{Var}\left(\varepsilon_{1} \mid \mathrm{X}_{1}\right)=1$. The class of weighted least squares estimators is defined as,

$$
\hat{\theta}_{n}(a)=\left\{\Sigma_{i} x_{i} x_{i}^{\prime} a_{i}^{-2}\right\}^{-1} \Sigma_{i} x_{i} Y_{i} a_{i}^{-2}
$$

where $\left\{a_{1}, 1 \leq \mathrm{i} \leq \mathrm{n}\right\}$ are suitable weights. Under regularity conditions, $\mathrm{n}^{1 / 2}\left[\hat{\theta} \mathrm{n}(\mathrm{a})-\boldsymbol{\theta}^{\circ}\right]^{\prime}$ 's limiting covariance matrix has lower Gauss-Markov bound $\Phi_{0}=\left\{E\left[X X^{\prime} \sigma^{-2}(X)\right]\right\}^{-1}$, and it is achieved by the unfeasible generalized least squares estimator $\hat{\theta}_{n}(\sigma)$, where $\sigma 1=\sigma\left(X_{1}\right)$. When the errors are conditionally normally distributed, $\Phi_{0}$ is the Cramer-Rao bound, but the normal model is never exactly true. In the presence of departures from the normality hypothesis, $\theta_{n}(\sigma)$ may be very inefficient. On the other hand outlying $X_{I}$ observations can adversely affect estimators such as (2.2). Therefore, it seems reasonable to consider estimators which bound the influence of the data.

Maronna and Yohai (1981) proved the asymptotic properties of a very general class of $M$-estimators of location and scale, implicitly defined as the simultaneous solution to,

$$
\sum_{\mathrm{i}} \phi\left(\mathrm{X}_{\mathrm{i}},\left(\mathrm{Y}_{\mathrm{i}}-\mathrm{X}_{\mathrm{i}}^{\prime} \theta\right) / \sigma\right) \mathrm{X}_{\mathrm{i}}=0 \text { and } \sum_{\mathrm{i}} \chi\left(\mathrm{X}_{\mathrm{i}},\left(\mathrm{Y}_{\mathrm{i}}-\mathrm{X}_{\mathrm{i}}^{\prime} \theta\right) / \sigma\right)=0 \text {, }
$$

where $E\left[\phi\left(X,\left(Y-X^{\prime} \theta^{\circ}\right) / \sigma\right) X \mid X\right]=0$ and $E\left[\chi\left(X,\left(Y-X^{\prime} \theta^{\circ}\right) / \sigma\right) \mid X\right]=0$. All known proposals for $\phi(.$.$) (see Hampel et. al 1986) may be written in the form,$

$$
\phi(X, r)=w(X) \psi(r \quad v(X))
$$

for appropriate functions $\psi: \mathbb{R} \rightarrow \mathbb{R}$ and weight functions $w: \mathbb{R}^{\mathrm{P}} \rightarrow \mathbb{R}^{+}$and $\mathrm{v}$ : $\mathbb{R}^{\mathbb{P}} \rightarrow \mathbb{R}^{+}$. The function $\psi($.$) bounds the influence of residuals and w($.$) and$ $v($.$) , the influence of leverage. Relles (1968) and Huber (1973), uses w(X)=1$, $v(X)=1$. There are a large number of $\psi$-function proposals. A popular proposal is the Huber's $\psi$-function, i.e. $\psi(u)=u \min \langle 1, c /|u|\}$, where $c\rangle 0$ is an appropriate chosen constant. In these cases, the corresponding M-estimators have bounded residual influence but the influence of leverage is unbounded. For a discussion on different choices of $w($.$) and v($.$) , see Hampel et. al$ (1986). Under conditional heteroskedasticity, estimators as (2.3) are not scale invariant. Carroll and Ruppert (1982) named $\ddot{\theta}(\sigma)$, the optimal $\mathrm{M}$-estimator (OME) under heteroskedasticity, where 
$Q_{n}\left\{\check{\theta}_{n}(a), a\right\}=0$,
and $Q_{n}\{\theta, a\}=\sum_{i} \phi\left(X_{i},\left(Y_{i}-X_{i}^{\prime} \theta\right) / a_{i}\right) X_{i}^{\prime} a_{i}$

The conditions for asymptotic normality of the OME are very similar to those given in Maronna and Yohai (1981). Let us introduce the following notation,

$\lambda(\Delta)=E\left\{X \quad \sigma(X)^{-1} \phi\left(X, \varepsilon-\Delta^{\prime} X \sigma(X)^{-1}\right)\right\}, L(X)=\sup _{u}|\phi(X, U)|\|X\|$ and

$\mathrm{H}(\mathrm{X})=\sup _{\mathrm{U}}\left|\phi^{\prime}(\mathrm{X}, \mathrm{U})\right|$ where $\phi^{\prime}(\mathrm{X}, \mathrm{U})=\partial \phi(\mathrm{X}, \mathrm{U}) / \partial \mathrm{U}$. We assume,

N1.- For each $\mathrm{X}, \phi(\mathrm{X},$.$) is odd, uniformly continuous, nondecreasing, \phi(\mathrm{X}, \mathrm{U})\rangle$

0 for $U>0$ and the conditional distribution of $\varepsilon$ is symmetric about zero.

N2. $-\operatorname{Pr}\{\sigma(X) \leq \delta\}=0$ for some $\delta>0$.

N3.- $\lambda(\Delta)$ has a nonsingular derivative at $0,-V(\sigma)$ say,

(that is $|\lambda(\Delta)-\lambda(0)+V(\sigma) \Delta|=0(\|\Delta\|)$ ).

N4.- $E\left\{H(X)\|X\|^{2} L(X)\right\} \infty$.

N5.- $E\left(H(X)\|X\|^{2}\right\}<\infty$.

N6. $-E\|X\|<\infty$.

N7. $-E\left\{L(X)^{2}\right\} \infty$.

Condition $N 1$ implies that $E\left\{\phi\left[X_{1}, \varepsilon_{1} S\left(X_{1}\right)\right] \mid X_{1}\right\}=0$ for any function $S\left(X_{1}\right)$ depending on $X_{1} i \geq 1$. Condition N2 is required by Robinson (1987) and Delgado (1989) for the adaptation proof of the semiparametric weighted least squares estimator (SWLSE). Alternatively, N2 may be removed by multiplying $\|\mathrm{X}\|$ by $\sigma(X)^{-1}$ in N4-N7. We have found convenient to set out the conditions in this way in order to make comparisons with conditions needed in the next section. Conditions N3-N7 are required by Maronna and Yohai (1981). N3 and N7 guarantee that the asymptotic covariance matrix of the OME is positive definite (p.d). .Note that for bounded influence estimators, as those with $\phi$-functions as $(2.4), L(X)$ is bounded. However, for classical M-estimators (i.e. $w(X)=v(X)=$ 1), N4 implies that $\mathrm{E}\|\mathrm{X}\|^{3}<\infty$ which seems a quite strong requirement. A normality proof in this case is possible, assuming $E\|X\|^{2}<\infty$, by using the results in Yohai and Maronna (1979). When, in (2.4), $\|\mathrm{X}\| \mathrm{w}(\mathrm{X}), \psi($.$) and \psi^{\prime}($. are bounded, N4-N7 holds if $E\|X\|^{2}<\infty$.

Theorem 1.- If N1-N7 hold,

$$
\mathrm{n}^{1 / 2}\left[\check{\theta}_{\mathrm{n}}(\sigma)-\theta^{o}\right] \stackrel{d}{\rightarrow} \mathrm{N}\left(0, \Phi_{1}\right)
$$

where, $\Phi_{1}=\mathrm{V}(\sigma)^{-1} \mathrm{E}\left[\phi(\mathrm{X}, \varepsilon)^{2} \mathrm{XX} / \sigma^{2}(\mathrm{X})\right] \mathrm{V}(\sigma)^{-1}$.

Proof.- See Appendix.

The purpose of this paper is to obtain estimators first order asymptotically equivalent to $\check{\theta}_{n}(\sigma)$. We propose to estimate $\sigma\left(\mathrm{X}_{1}\right)$ by nonparametric regression.

\section{3.- Bounded Influence Function Semiparametric Estimators}

When the functional form of $\sigma\left(\mathrm{XI}_{\mathrm{I}}\right)$ is known and a preliminary root-n-consistent estimator of $\theta^{o}, \tilde{\theta}_{n}$ say, is available, consistent estimators of 
$\sigma^{2}\left(X_{1}\right)=\left[\sigma\left(X_{1}\right)\right]^{2}$ are obtained by regressing ( $\left.Y_{1}-X_{i}^{\prime} \tilde{\theta}_{n}\right)^{2}$ against the known design of $\sigma^{2}\left(\mathrm{X}_{1}\right)$. Under regularity conditions, the corresponding weighted least squares estimators (WLSE) of $\theta^{\circ}$ are as first order efficient as $\hat{\theta}_{n}(\sigma)$.

Carroll and Ruppert (1982) suggested the use the of consistent parametric weights to construct robust estimators such as those defined in (2.5) but using $\mathrm{w}(\mathrm{X})=\mathrm{v}(\mathrm{X})=1$ and $\mathrm{X}$ fixed. They proved that these estimators are, under regularity conditions, asymptotically equivalent to On$_{n}(\sigma)$. These estimators are nonrobust with respect to the assumed parameterization of $\sigma^{2}(X)$.

Rose (1978) proposed several nonparametric estimators of $\sigma^{2}\left(X_{1}\right)$. One of them is obtained by performing a nonparametric regression of $\left(Y_{1}-X_{1} \tilde{\theta}_{n}\right)^{2}$ against the regressors on which $\sigma^{2}\left(X_{1}\right)$ is known to depend. Carroll (1982) and Robinson (1987) proved, under different regularity conditions, that the corresponding SWLSE asymptotically achieves the Gauss-Markov bound. Carroll (1982) used kernel regression while Robinson (1987) used k nearest neighbors $(k-n n)$ regression.

In this paper we follow Robinson's approach. If $\sigma(X)$ is known to depend on a subset $\mathrm{dxl}(\mathrm{d} \leq \mathrm{p})$ vector $\mathrm{X}_{1}=\left(\mathrm{X}_{11}, \ldots, \mathrm{X}_{\mathrm{d} 1}\right)$ ' of nondegenerate elements of $\mathrm{X}_{1}$, and given a positive integer $k=k(n)$, the sequence of $k-n n$ nonparametric weights $\left\{\omega_{1} \mathrm{j}(\mathrm{k}), \mathrm{i}, \mathrm{j}=1, \ldots, n\right\}$ is defined by

$$
\omega_{i j}(k)=1(i \neq j) r_{i j}^{-1} \quad \sum_{\tau=p_{1 j}}^{p_{1 j}^{+} r_{1 j}-1} c_{\tau}(k) \quad 1 \leq i, j \leq n
$$

where for $1 \leq \mathrm{i} \leq \mathrm{n}, \mathrm{c}_{\mathrm{i}}(\mathrm{k})>0,1 \leq \mathrm{i} \leq \mathrm{k} ; \mathrm{c}_{\mathrm{i}}(\mathrm{k})=0 \mathrm{i}>\mathrm{k} ; \sum_{\mathrm{i}=1}^{\mathrm{k}} \mathrm{c}_{\mathrm{i}}(\mathrm{k})=1$, and

$$
\mathrm{p}_{\mathrm{ij}}=1+\sum_{\mathrm{t}} 1\left(\rho_{\mathrm{it}}<\rho_{\mathrm{ij}}\right) ; \quad \mathrm{r}_{\mathrm{ij}}=1+\sum_{\mathrm{t}} 1\left(\rho_{\mathrm{it}}=\rho_{\mathrm{ij}}\right) \quad \mathrm{i} \neq \mathrm{j}
$$

where the sums are over $1 \leq t \leq n, t \neq i, j$ and $1($.$) is the indicator function.$

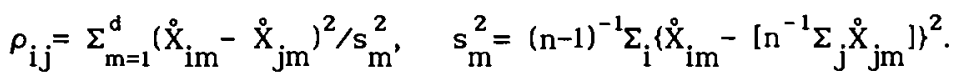

The uniform weights $\mathrm{cl}(\mathrm{k})=\mathrm{k}^{-1}, \mathrm{i}=1, \ldots, \mathrm{k}$, satisf $\mathrm{y}$ these conditions. Other weights satisfying these conditions can be found in Stone (1977). These $\mathrm{k}-\mathrm{nn}$ weights do not use the own observation. This sample splitting is not required for the consistency of the $k-n n$ weights but it is technically convenient in semiparametric estimation. This sample splitting technique has also been employed by Robinson (1987) and Delgado (1989). Given a preliminary root-n-consistent estimator of $\theta^{\circ}$, $\tilde{\theta}_{n}$ say, a consistent estimate of $\sigma_{i}^{2}$ is,

$$
\hat{\sigma}_{i}^{2}=\sum_{j}\left(Y_{j}-X_{j}^{\prime} \tilde{\theta}_{n}\right)^{2} \omega_{i j}(k) \text {. }
$$

We suggest estimating $\theta^{\circ}$ by the semiparametric weighted M-estimator (SWME), $\check{\theta}_{n}(\hat{\sigma})$ where $\hat{\sigma}_{1}=\left[\hat{\sigma}_{1}^{2}\right]^{1 / 2}$. Unlike $\hat{\theta}_{n}(\sigma)$ and $\hat{\theta}_{n}(\hat{\sigma}), \check{\theta}_{n}(\hat{\sigma})$ has bounded influence function.

One would expect that the higher order efficiency of $\check{\theta}_{n}(\hat{\sigma})$ will improve by using an iterative procedure (i.e. computing new $\hat{\sigma}_{1}$ at each iteration). A full iterated SWLSE is obtained at once by using the following pure nonparametric estimator of $\sigma^{2}\left(X_{i}\right)$,

$$
\tilde{\sigma}_{i}^{2}=\sum_{j} Y_{j}^{2} \omega_{i j}(k)-\left\langle\sum_{j} Y_{j} \omega_{i j}(k)\right\}^{2},
$$


Delgado (1989) proved, under Robinson (1987) regularity conditions, that

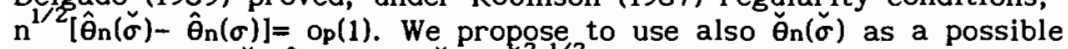
alternative to $\breve{\theta}_{\mathrm{n}}(\hat{\sigma})$ where $\check{\sigma}_{1}=\left[\hat{\sigma}_{1}^{2}\right]^{1 / 2}$.

\section{4.- Asymptotic Theory}

Robinson (1987) noted that it is technically convenient to relate the moment conditions on $(\mathrm{Y}, \mathrm{X})$ and the rate of convergence of $\mathrm{k}$. We assume that,

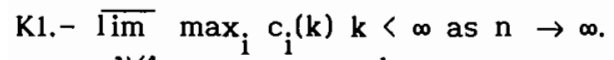

K2. $-k^{-v / 4} n \rightarrow 0$ and $n^{-1} k \longrightarrow 0$ as $n \rightarrow \infty$ for $u>4$.

Besides N1-N3, we need stronger moment conditions in N4-N7. In particular for the $v$ in $\mathrm{K} 2$ we need,

R1. - (a) $E\left|H(X)\|X\|^{2} L(X)\right|^{v /(U-2)}<\infty$ and (b) $E|H(X)\|X\| L(X)| \varepsilon \sigma(X)||^{v /(U-2)}<\infty$. R2. - (a) $E\left|H(X)\|X\|^{\mathbb{Q}}\right|^{U /(U-2)}<\infty$ and (b) $E|H(X)\|X\|| \varepsilon \sigma(X)||^{U /(U-2)}<\infty$.

R3. $-E\|X\|^{2 v /(v-2)}<\infty$.

R4. - $E|L(X)|^{2 U /(U-2)}<\infty$.

R5. $-E|\varepsilon \sigma(X)|^{v}<\infty$.

The difference between assumptions $\mathrm{N}$ and $\mathrm{R}$ are similar as in the weighted least squares estimator case. The asymptotic normality of $\hat{\theta}_{n}(\sigma)$ needs that $\mathrm{E}\|\mathrm{X}\|^{2}<\infty$ and the SWLSE of Robinson (1987) needs $\mathrm{E}\|\mathrm{X}\|^{2 v /(U-2)}<\infty$. This last condition implies R1-R4 when, in (2.4), $w(X)\|X\|, \psi($.$) and \psi^{\prime}($.$) are bounded.$ R3 and R5 are needed in Robinson (1987), Newey (1987) and Delgado (1989).

Theorem 2.- If N1-N3, K1, K2 and R1-R5 hold:

(a)

$$
\mathrm{n}^{1 / 2}\left[\check{\theta}_{\mathrm{n}}(\hat{\sigma})-\theta^{\circ}\right] \stackrel{\mathrm{d}}{\rightarrow} \mathrm{N}\left(0, \Phi_{1}\right) \text {, }
$$

(b)

$$
\mathrm{n}^{1 / 2}\left[\check{\theta}_{\mathrm{n}}(\check{\sigma})-\theta^{\circ}\right] \stackrel{\mathrm{d}}{\rightarrow} \mathrm{N}\left(0, \Phi_{1}\right) \text {. }
$$

Proof.- See Appendix.

Maronna and Yohai (1981) recommended to construct interval estimators, estimating the asymptotic variance by its natural sample analog. In our case, $\Phi_{1}$ is estimated by,

$$
\hat{\Phi}_{1}=\hat{V}_{n}(\hat{\sigma})^{-1} n^{-1} \sum_{i} X_{i} X_{i}^{\prime} \hat{\sigma}_{i}^{-2} \phi^{2}\left(X_{i},\left[Y_{i}-X_{i}^{\prime} \check{\theta}_{n}(\hat{\sigma})\right] \hat{\sigma}_{i}^{-1}\right) \hat{V}_{n}(\hat{\sigma})^{-1}
$$

where $\hat{V}_{n}(\hat{\sigma})=n^{-1} \Sigma_{i} X_{i} X_{i}^{\prime} \hat{\sigma}_{i}^{-2} \phi^{\prime}\left(X_{i},\left[Y_{i}-X_{i}^{\prime} \check{\theta}_{n}(\hat{\sigma})\right] \hat{\sigma}_{i}^{-1}\right)$.

We can substitute $\hat{\sigma}_{1}$ by $\check{\sigma}_{1}$ in (4.1).

\section{5.- Monte Carlo.}

The experiments follow the model

$$
Y_{i}=\theta_{1}^{0}+\theta_{2}^{o} X_{i}+\varepsilon_{i} \sigma\left(X_{i}\right), i \geq 1
$$


with $\theta_{1}^{\circ}=\theta_{2}^{\circ}=1$. and $X_{1} \sim$ iid Uniform $(0,2)$. The $\varepsilon_{1}^{\prime}$ s were generated iid and independent of $X_{i}$ as follows,

CONTAMINATED. $-\varepsilon_{\mathrm{i}} \sim$ iid $\{.9 \mathrm{~N}(0,1)+.1 \mathrm{~N}(0,9)\} / \sqrt{1.8}$.

NORMAL. $-\varepsilon_{i}$ iid $N(0,1)$.

Note that $\operatorname{Var}(\varepsilon)=1$ in all models. The residual conditional variances are constructed according to the models,

$$
\begin{aligned}
& \text { Model 1. }-\sigma\left(X_{i}\right)=\exp \left(\gamma X_{i}\right) \\
& \text { Model 2. }-\sigma\left(X_{i}\right)=|1 .+X|^{\delta} .
\end{aligned}
$$

We have only used uniform weights; i.e. in $(3.1), \mathrm{cl}=1 / \mathrm{k}$ for all $\mathrm{i} \leq \mathrm{k}$. We report two choices of $k, \hat{\theta}_{n}(\hat{\sigma})^{1}, \hat{\theta}_{n}(\check{\sigma})^{1}, \hat{\theta}_{n}(\hat{\sigma})^{1}, \hat{\theta}_{n}(\check{\sigma})^{1}$ are computed with $k=$ $\left[n^{/ 2}\right]$, while $\hat{\theta}_{n}(\hat{\sigma})^{2}, \hat{\theta}_{n}(\hat{\sigma})^{2}, \hat{\theta} n(\hat{\sigma})^{2}, \hat{\theta}_{n}(\dot{\sigma})^{2}$ are computed with $k=\left[n^{2 / 3}\right]$.

We compare the unfeasible estimator $\hat{\theta}_{n}(\sigma)$, the OLSE $\hat{\theta}_{n}(1)$, the SWLSE's $\hat{\theta}_{n}(\hat{\sigma})$ and $\hat{\theta}_{n}(\check{\sigma})$, the unfeasible $\operatorname{OME}_{\breve{\theta}}(\sigma)$, the M-estimator with fixed scale $\check{\theta}_{n}(1)$ and the SWME's $\breve{\theta}_{n}(\hat{\sigma})$ and $\breve{\theta}_{n}(\check{\sigma})$. In order to save space we only report results for the slope coefficient. We have only considered the classical Huber's estimator, i.e. $\psi(U)=U \min \{1, c /|U|\}$ in $(2.4)$, where we choose $c=1.345$. The robust estimators were computed using reweighted least squares. This $\mathrm{c}$ produces, under normality, $\theta_{n}(\sigma) 95 \%$ as efficient as $\hat{\theta}_{n}(\sigma)$. We also report results for the least absolute deviation estimator (LADE) without scaling. . This estimator is used as the starting point in the reweighted procedure for the Huber's estimators and for the estimation of the residuals in order to compute $\hat{\sigma}$.

The tables show the bias (BIAS), variance (VAR) and the relative efficiency (EFF) of the different estimators for sample sizes of $n=30, n=100$ and $n=500$ with $10,000,5,000$ and 1,000 replications respectively. The efficiency is the ratio of the mean square error (MSE) of the estimator with respect to MSE of $\hat{\theta}_{n}(\sigma)$. We report results for the different disturbances and the heteroskedasticity models 1 and 2 with different parameter values for $\delta$ and $\gamma$ which produce different degrees of heteroskedasticity. All the programs were written in FORTRAN-77 double precision and NAG-13 routines were used to generate the variates. The programs were run on the Indiana University VAXes.

The simulations strongly support the applicability of our theorem.

Through the experiments, the SWME's are always more efficient than the SWLSE's. We observe important gains in efficiency of the semiparametric estimates as the sample size increases while the EFF of the others estimators is not significantly affected. It is obviously due to the fact that the nonparametric estimates of the residual variances become more accurate as the sample size increases. This also happens when working with parametric weights. Therefore, for the smallest sample size $(n=30)$ and when the heteroskedasticity is mild, the SWME's are, sometimes, more inefficient than the LADE. However, when $n=100$ or 500, the SWME's EFF is typically greater than one under departures from normality. Samples sizes of 30 or 100 are small in a cross-section context where samples of several thousands of observations are common. In the normal case, the SWME's EFF is quite close to their asymptotic values when $n=500$ and always is greater than the SWLSE's EFF. It is observed, in general, that for similar degrees of 
heteroskedasticity, the semiparametric estimators based on $\hat{\sigma}_{1}$ behaves slightly better than those based on $\sigma_{1}$ when the conditional variances are small, while the latter perform better than the former when the conditional variances are larger. The choice of $\mathbf{k}$ does not seem to affect significantly the results. We proceed to discuss the tables in some detail.

Table 1 shows results for CONTAMINATED errors. Note that the contamination is quite moderate. Simulations using this distribution have been also reported by Huber (1973) and Carroll and Ruppert (1982). We have considered MODEL 1 and MODEL 2 with $\gamma=0,-1,-2$ and $\delta=1,35$. So, in MODEL 1 we have small variances while in MODEL 2 the variances are larger. The severity of the heteroskedasticity is comparable in the two models when $\gamma=-1,-2$ and $\delta=3,5$. When $n=500$ the semiparametric estimators efficiencies are fairly close to their asymptotic values. However, when $n=30$, the semiparametric estimators are more inefficient than the LADE and OLSE, in some cases, especially when the heteroskedasticity is mild. When the heteroskedasticity is heavy, i.e. $\gamma=$ -2 and $\delta=5$, the SWLSE's are more efficient than than the OLSE for $n=30$, but they are more inefficient than the LADE in MODEL 1 (when $\gamma=-2$ ). As expected, the SWME's are always more efficient than the SWLSE's. However, the SWME's are generally more inefficient than the LADE for $n=30$ in MODEL 1. In MODEL 2, with $\mathrm{n}=30$, the SWME's appear to be more inefficient than the LADE only when $\delta=0,1$ and $k=\left[n^{1 / 2}\right]$. When $n=100$, the SWLSE's are always more efficient than the OLSE but the SWLSE's are still more inefficient than the LADE in MODEL 1 and MODEL 2 for $\delta=1,3$. For $n=100$, the SWME's are always more efficient than LADE, OLSE and SWLSE's. They, some times, appear to be more efficient than $\hat{\theta}_{\mathrm{n}}$ (e.g. $\gamma=0,-1, \delta=3$ ), as the asymptotic theory predicts. This prediction is fully supported when $n=500$. In this case, the SWME's EFF is always greater than 1 and the SWLSE's EFF is closer to 1 , but they still behaves worse than the LADE when $\gamma$ and $\delta$ are small. We have tried other distributions (e.g.

-Student and Laplace) with similar results.

In Table 2 we report results for NORMAL errors with MODEL $1(\gamma=-2)$ and MODEL $2(\delta=5)$. When $n=30$, the LADE is more efficient than $\check{\theta}_{n}(\dot{\sigma})$ and $\hat{\theta}_{n}(\check{\sigma})$ in "MODEL 1. In the other cases, the SWME's are more efficient than LADE. As the sample size increases, the SWME's EFF approaches their asymptotic value (.95). Note that for $n=500$, the SWME's EFF are closer to their asymptotic value than the SWLSE's EFF. In fact the SWME's appear to be more efficient than the SWLSE's in all cases reported, though asymptotically it is $5 \%$ more inefficient. this fact is of practical importance. In Tables 3 and 4 we report results for LAPLACE and T4 errors. The OME's EFF is smaller, in these cases, than in the CONTAMINATED case and, therefore, the SWME's when $n=500$ are less spectacular. However, the differences in EFF between the SWME's and SWLSE's are larger than in the CONTAMINATED case. For $n=30$, the SMWE's typically perform better than the LADE and always perform better than the SWLSE's.

\section{Conclusions}

We have seen that the introduction of robust methods in the estimation of semiparametric models is of practical relevance. The Monte Carlo reported shows that our method can be widely used, without great losses in efficiency when the data are close to normal but heteroskedastic. 


\section{APPENDIX}

Proof of Theorem 1: In proving the theorem we do not need to assume that $\check{\theta}_{n}(\sigma)$ uniquely solves $Q_{n}(\theta, \sigma)=0$ but rather that $\left\|Q_{n}\left(\check{\theta}_{n}(\sigma), \sigma\right)\right\|$ is majorized by twice the infimum of $\left\|Q_{n}(\theta, \sigma)\right\|$ over $\theta$. Let define,

$$
U_{n}(\Delta, a)=n^{-1 / 2} \sum_{i} \phi\left(X_{i},\left(\varepsilon_{i}-n^{-1 / 2} \Delta^{\prime} X_{i} \sigma_{i}^{-1}\right) \sigma_{i} a_{i}^{-1}\right) X_{i} a_{i}^{-1}
$$

Since $Q_{n}\left(\check{\theta}_{n}(\sigma), \sigma\right)=U_{n}\left(n^{1 / 2}\left(\check{\theta}_{n}(\sigma)-\theta^{0}\right), \sigma\right)=0$,

where

$$
\left\|U_{n}\left(n^{1 / 2}\left(\check{\theta}_{n}(\sigma)-\theta^{\circ}\right), \sigma\right)\right\| \leq 2\left\|U_{n}\left(\Delta^{*}, \sigma\right)\right\| \text {, }
$$

$$
\Delta^{*}=-V(\sigma)^{-1} U_{n}(0, \sigma)=o_{p}(1),
$$

by N3 and since by Chevyshev's inequality $U_{n}(0, \sigma)=O_{p}\left\{\left[E\|U(O, \sigma)\|^{2}\right]^{1 / 2}\right\}=$ $\mathrm{Op}(1)$ by $\mathrm{N} 1$ and N7. Then noting that, by the Lindeberg-Levy central limit theorem (clt), $\Delta^{*} \stackrel{d}{\rightarrow} N\left(O, \Phi_{1}\right)$, the theorem follows from (a.2) af ter establishing that,

$$
\begin{gathered}
\left\|U_{n}\left(\Delta^{*}, \sigma\right)\right\|=o_{p}(1), \\
n^{1 / 2}\left(\check{\theta}_{n}(\sigma)-\theta^{\circ}\right)=o_{p}(1) .
\end{gathered}
$$

We conclude (a.4) from (a.3) and

$$
\sup _{\|\Delta\| \leq M}\left\|U_{n}(\Delta, \sigma)-U_{n}(0, \sigma)+V(\sigma) \Delta\right\|=o_{p}(1) .
$$

(a.6) follows from N3 and,

$$
\sup _{\|\Delta\| \leqslant M}\left\|U_{n}(\Delta, \sigma)-U_{n}(0, \sigma)-E\left\{U_{n}(\Delta, \sigma)\right\}\right\|=o_{p}(1) \text {. }
$$

In order to prove (a.7), take for convenience $M=1$. For $0<\delta<1$ define,

$$
\mathrm{S}_{\mathrm{n}}(\Delta, \sigma)=\sup _{\left\|\Delta^{1}-\Delta\right\| \leq \delta} \quad\left\|\mathrm{U}_{\mathrm{n}}\left(\Delta^{1}, \sigma\right)-\mathrm{U}_{\mathrm{n}}(\Delta, \sigma)\right\|
$$

Since for each fixed $\delta$ we can cover the ball of radius 1 in $\mathbb{R}^{p}$ by a finite number of balls of radius $\delta$, we conclude (a.7) from,

$$
U_{n}(\Delta, \sigma)-U_{n}(0, \sigma)-E\left\{U_{n}(\Delta, \sigma)-U_{n}(0, \sigma)\right\}=o_{p}(1)
$$

for each fixed $\Delta$, and for all $0<\delta<1$, all $\mathrm{n}$ and all $\|\Delta\| \leq 1$,

$$
\begin{gathered}
E\left\{S_{n}(\Delta, \sigma)\right\} \leq \delta O(1), \\
S_{n}(\Delta, \sigma)-E\left\{S_{n}(\Delta, \sigma)\right\}=o_{p}(1),
\end{gathered}
$$

(a.9) follows from, 


$$
\begin{aligned}
\operatorname{Var}\left\{U_{n}(\Delta, \sigma)-U_{n}(0, \sigma)\right\} & =n^{-1} \sum_{i} \operatorname{Var}\left\|X_{i} \sigma_{i}^{-1} \phi\left(X_{i},\left(\varepsilon_{i}-n^{-1 / 2} \Delta^{\prime} X_{i} \sigma_{i}^{-1}\right)\right)\right\| \\
\leq & n^{-1} \sum_{i} E\left\|X_{i} \sigma_{i}^{-1} \phi\left(X_{i},\left(\varepsilon_{i}-n^{-1 / 2} \Delta^{\prime} X_{i} \sigma_{i}^{-1}\right)\right)\right\|^{2} \\
& \leq n^{-1 / 2} E\left\{\sigma(X)^{-2}\|X\|^{2} H(X) L(X)\right\}=O\left(n^{-1 / 2}\right),
\end{aligned}
$$

applying a mean value theorem (mvt) argument, N2 and N4. (a.10) follows from

$$
E\left\{S_{n}(\Delta, \sigma)\right\} \leq \delta E\left\{\sigma(X)^{-2}\|X\|^{2} H(X)\right\} \leq \delta O(1),
$$

by N2 and N5. (a.11) is proved using similar arguments as in (a.9), in particular,

$$
\operatorname{Var}\left\{S_{n}(\Delta, \sigma)\right\} \leq E\left\{S_{n}^{2}(\Delta, \sigma)\right\} \leq \delta n^{-1 / 2} E\left\{\sigma(X)^{-2}\|X\|^{2} H(X) L(X)\right\}=O\left(n^{-1 / 2}\right) .
$$

(a.4) holds if for each $\eta>0, \tau>0$ and $M$, there exists a M1 satisfying,

$$
\operatorname{Pr}\left\{\inf _{\|\Delta\| \leq M_{1}}\left\|U_{n}(\Delta, \sigma)\right\|>\eta\right\}>1-\tau,
$$

and (a.12) follows from(a.6) using same arguments as Jureckova's (1977) proof of her Lemma 5.2.

Proof of Theorem 2 (a): Let introduce the following notation,

$$
\tilde{\sigma}_{i}^{2}=\sum_{j}\left(Y_{j}-X_{j}^{\prime} \tilde{\theta}_{n}\right)^{2} \omega_{i j} \text { and } \bar{\sigma}_{i}^{2}=\sum_{j} \sigma_{j}^{2} \omega_{i j}
$$

where $\omega_{i j}=\omega_{i j}(k)$. We need the following Lemmas, proved in Robinson (1987),

Cemma 1.- Let $f($.$) be a Borel function such that E|f(x)|^{p}<\infty$,

$$
E\left\{\sum_{i}\left|f\left(x_{i}\right)-f\left(X_{1}\right)\right|{ }^{p} \omega_{1 i}\right\}=o(1) \text {. }
$$

Lemma 2.- $\operatorname{Pr}\left\{\min _{\mathrm{i}} \sigma_{\mathrm{i}} \leq \delta\right\}=0$ all $\mathrm{n}$ and some $\delta>0$.

Lemma 3. $\operatorname{Pr}\left\{\bar{\sigma}_{1} \leq \delta\right\}=0$ all $\mathrm{n}$ and some $\delta>0$.

Lemma 4.- $\operatorname{Pr}\left\{\min _{\mathrm{i}} \bar{\sigma}_{i} \leq \delta\right\}=0$ all $\mathrm{n}$ and some $\delta>0$.

Lemma 5. $-\left\{\min _{i} \tilde{\sigma}_{i}^{2}\right\}^{-1}=o_{p}(1)$.

Lemma 6.- $\left\{\min _{i} \hat{\sigma}_{i}^{2}\right\}^{-1}=o_{p}(1)$.

Lemma 7.- $\sum_{i}\left|\hat{\sigma}_{i}^{2}-\tilde{\sigma}_{i}^{2}\right|^{2}=o_{p}\left(k^{-1}\right)$.

Lemma 8. $-E\left\{\left|\tilde{\sigma}_{1}^{2}-\bar{\sigma}_{1}^{2}\right|^{v / 2}\right\}=O\left(k^{-v / 4}\right)$.

Lemma 9.- $\max _{i}\left|\hat{\sigma}_{i}^{2}-\tilde{\sigma}_{i}^{2}\right|=o_{p}\left(k^{-1 / 2}\right)$. 
Lemma 10.- $\max _{i}\left|\tilde{\sigma}_{i}^{2}-\bar{\sigma}_{i}^{2}\right|=o_{p}\left(n k^{-v / 4}\right)$.

We foliow the same strategy of proof as in Theorem 1. We first prove that,

$$
\sup _{\|\Delta\| \leq M}\left\|U_{n}(\Delta, \hat{\sigma})-U_{n}(0, \hat{\sigma})+V(\sigma) \Delta\right\|=o_{p}(1) .
$$

We conclude (b.1) from (a.6) and,

$$
\sup _{\|\Delta\| \leq M}\left\|U_{n}(\Delta, \hat{\sigma})-U_{n}(0, \hat{\sigma})-\left\{U_{n}(\Delta, \sigma)-U_{n}(0, \sigma)\right\}\right\|=o_{p}(1) .
$$

(b.2) follows from,

$$
\begin{aligned}
& \sup _{\|\Delta\| \leq M}\left\|n^{-1 / 2} \sum_{i} X_{i}\left(\hat{\sigma}_{i}^{-1}-\tilde{\sigma}_{i}^{-1}\right) R_{i}(\Delta, \hat{\sigma})\right\|=o_{p}(1), \\
& \sup _{\|\Delta\| \leq M}\left\|\mathrm{n}^{-1 / 2} \sum_{\mathrm{i}} \mathrm{X}_{\mathrm{i}}\left(\tilde{\sigma}_{\mathrm{i}}^{-1}-\bar{\sigma}_{\mathrm{i}}^{-1}\right) \mathrm{R}_{\mathrm{i}}(\Delta, \hat{\sigma})\right\|=o_{\mathrm{p}}(1) \text {, } \\
& \sup _{\|\Delta\| \leq M}\left\|n^{-1 / 2} \sum_{i} X_{i}\left(\bar{\sigma}_{i}^{-1}-\sigma_{i}^{-1}\right) R_{i}(\Delta, \hat{\sigma})\right\|=o_{p}(1), \\
& \sup _{\|\Delta\| \leq M}\left\|\mathrm{n}^{-1 / 2} \sum_{\mathrm{i}} \mathrm{X}_{\mathrm{i}} \sigma_{i}^{-1}\left[\mathrm{R}_{\mathrm{i}}(\Delta, \hat{\sigma})-\mathrm{R}_{\mathrm{i}}(\Delta, \tilde{\sigma})\right]\right\|=o_{\mathrm{p}}(1) \text {, } \\
& \sup _{\|\Delta\| \leq M}\left\|n^{-1 / 2} \sum_{i} X_{i} \sigma_{i}^{-1}\left[R_{i}(\Delta, \tilde{\sigma})-R_{i}(\Delta, \bar{\sigma})\right]\right\|=o_{p}(1), \\
& \sup _{\|\Delta\| \leq M}\left\|n^{-1 / 2} \sum_{i} x_{i} \sigma_{i}^{-1}\left[R_{i}(\Delta, \sigma)-R_{i}(\Delta, \sigma)\right]\right\|=o_{p}(1) \text {, }
\end{aligned}
$$

$K \mathrm{n}^{-1} \sum_{\mathrm{i}}\left\|\mathrm{X}_{\mathrm{i}}\right\|^{2} \mathrm{H}\left(\mathrm{X}_{\mathrm{i}}\right) \hat{\sigma}_{\mathrm{i}}^{-1}\left|\hat{\sigma}_{\mathrm{i}}^{-1}-\tilde{\sigma}_{\mathrm{i}}^{-1}\right| \leq$

by Lemmas 5,6 and 9 and $\mathrm{R} 1$ (where, henceforth, $\mathrm{K}$ is a generic constant). The left hand side of (b.4) is bounded by,

$K n^{-1} \sum_{i}\left\|X_{i}\right\|^{2} H\left(X_{i}\right) \hat{\sigma}_{i}^{-1}\left|\tilde{\sigma}_{i}^{-1}-\bar{\sigma}_{i}^{-1}\right| \leq$

$K \max _{i}\left|\tilde{\sigma}_{i}^{2}-\bar{\sigma}_{i}^{2}\right|\left\{\min _{i} \hat{\sigma}_{i} \min _{i} \tilde{\sigma}_{i} \min _{i} \bar{\sigma}_{i}\left(\min _{i} \tilde{\sigma}_{i}+\min _{i} \bar{\sigma}_{i}\right)\right\}^{-1} n^{-1} \sum_{i}\left\|X_{i}\right\|^{2} H\left(x_{i}\right)$

$=o_{p}\left(\mathrm{n} \mathrm{k}^{-v / 4}\right)$,

by Lemmas $4,5,6$ and 10 and R1. The left hand side of (b.5) is bounded by, $\left\{\min _{i} \hat{\sigma}_{i} \min _{i} \sigma_{i} \min _{i} \bar{\sigma}_{i}\left(\min _{i} \sigma_{i}+\min _{i} \bar{\sigma}_{i}\right)\right\}^{-1} \mathrm{Kn}^{-1} \sum_{i}\left\|X_{i}\right\|^{2} H\left(X_{i}\right)\left|\bar{\sigma}_{i}^{2}-\sigma_{i}^{2}\right|=o_{p}(1)$,

by Lemmas 2,3 and 6 and Markov's inequality, noting that, by Holder's inequality. 


$$
E\left\{\left\|X_{1}\right\|^{2} H\left(X_{1}\right)\left|\bar{\sigma}_{1}^{2}-\sigma_{1}^{2}\right|\right\} \leq\left\{E\left|\left\|X_{1}\right\|^{2} H\left(X_{1}\right)\right|^{v /(v-2)}\right\}^{(v-2) / v}\left\{E\left|\bar{\sigma}_{1}^{2}-\sigma_{1}^{2}\right|^{v / 2}\right\}^{2 / v}=o(1)
$$

by Lemma 1 and RI.(b.6), (b.7) and (b.8) follow by using same arguments as in (b.3), (b.4) and (b.5), after bounding the former expressions using a mvt argument. Next we prove that,

$$
\sup _{\|\Delta\| \leq M}\left\|U_{n}(\Delta, \hat{\sigma})-U_{n}(0, \sigma)+V(\sigma) \Delta\right\|=o_{p}(1)
$$

We conclude (b.9) from (b.l) and,

(b.10) follows from,

$$
\left\|U_{n}(0, \hat{\sigma})-U_{n}(0, \sigma)\right\|=o_{p}(1)
$$

$$
\begin{aligned}
& \left\|\mathrm{n}^{-1 / 2} \sum_{\mathrm{i}} \mathrm{x}_{\mathrm{i}}\left(\bar{\sigma}_{\mathrm{i}}^{-1}-\sigma_{\mathrm{i}}^{-1}\right) \phi\left(\mathrm{x}_{\mathrm{i}}, \varepsilon_{\mathrm{i}}\right)\right\|=o_{\mathrm{p}}(1),
\end{aligned}
$$

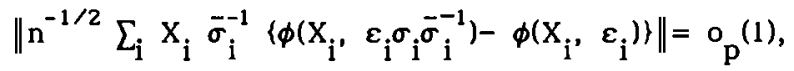

$$
\begin{aligned}
& \left\|\mathrm{n}^{-1 / 2} \sum_{\mathrm{i}} \mathrm{x}_{\mathrm{i}}\left(\hat{\sigma}_{\mathrm{i}}^{-1}-\tilde{\sigma}_{\mathrm{i}}^{-1}\right) \phi\left(\mathrm{x}_{\mathrm{i}}, \varepsilon_{\mathrm{i}} \sigma_{\mathrm{i}} \bar{\sigma}_{\mathrm{i}}^{-1}\right)\right\|=o_{\mathrm{p}}(1), \\
& \left\|\mathrm{n}^{-1 / 2} \sum_{\mathrm{i}} \mathrm{x}_{\mathrm{i}}\left(\tilde{\sigma}_{\mathrm{i}}^{-1}-\bar{\sigma}_{\mathrm{i}}^{-1}\right) \phi\left(\mathrm{x}_{\mathrm{i}}, \varepsilon_{\mathrm{i}} \sigma_{\mathrm{i}} \bar{\sigma}_{\mathrm{i}}^{-1}\right)\right\|=o_{\mathrm{p}}(1), \\
& \left\|n^{-1 / 2} \Sigma_{i} X_{i} \hat{\sigma}_{i}^{-1}\left\langle\phi\left(X_{i}, \varepsilon_{i} \sigma_{i} \hat{\sigma}_{i}^{-1}\right)-\phi\left(X_{i}, \varepsilon_{i} \sigma_{i}^{-\sigma_{i}^{-1}}\right)\right\}\right\|=o_{p}(1) .
\end{aligned}
$$

Using the fact that $\phi(\mathrm{X},$.$) is odd and \varepsilon$ is conditionally symmetric about zero, (b.11) follows from Chevyshev's inequality since,

$$
\begin{gathered}
\mathrm{E}\left\|\mathrm{n}^{-1 / 2} \sum_{\mathrm{i}} \mathrm{X}_{\mathrm{i}}\left(\bar{\sigma}_{\mathrm{i}}^{-1}-\sigma_{\mathrm{i}}^{-1}\right) \phi\left(\mathrm{X}_{\mathrm{i}}, \varepsilon_{\mathrm{i}}\right)\right\|^{2}=\mathrm{E}\left\{\left\|\mathrm{X}_{1}\right\|^{2}\left|\bar{\sigma}_{1}^{-1}-\sigma_{1}^{-1}\right|^{2} \phi\left(\mathrm{X}_{1}, \varepsilon_{1}\right)^{2} \|\right\} \\
\leq \mathrm{KE} \mathrm{E}\left(\mathrm{L}\left(\mathrm{X}_{1}\right)^{2}\left|\bar{\sigma}_{1}^{2}-\sigma_{1}^{2}\right|\right\},
\end{gathered}
$$

by N2, Lemma 3 and 4; and by Holder's inequality,

$E\left(L\left(X_{1}\right)^{2}\left|\bar{\sigma}_{1}^{2}-\sigma_{1}^{2}\right|\right\} \leq\left\{E\left|L\left(X_{1}\right)\right|^{2 v /(v-2)}\right\}^{(v-2) / v} E\left(\left|\bar{\sigma}_{1}^{2}-\sigma_{1}^{2}\right|^{v / 2}\right\}^{2 / v}=o_{p}(1)$,

by Lemma 1 and R4.We conclude (b.12) from Chevyshev's inequality, since, by triangle inequality, Lemma 3 and 4 and Holder's inequality,

$E\left\|n^{-1 / 2} \sum_{i} X_{i} \bar{\sigma}_{i}^{-1}\left\{\phi\left(X_{i}, \varepsilon_{i} \sigma_{i} \bar{\sigma}_{i}^{-1}\right)-\phi\left(X_{i}, \varepsilon_{i}\right)\right\}\right\|^{2} \leq$

$E\left|\left\|X_{1}\right\|^{2} \bar{\sigma}_{1}^{-2}\left\{\phi\left(X_{1}, \varepsilon_{1} \sigma_{1} \bar{\sigma}_{1}^{-1}\right)-\phi\left(X_{1}, \varepsilon_{1}\right)\right\}^{2}\right|=$

$E \mid\left\|X_{1}\right\|^{2} \bar{\sigma}_{1}^{-2}\left(\bar{\sigma}_{1}^{-1}-\sigma_{1}^{-1}\right) \varepsilon_{1} \sigma_{1}\left\{\phi\left(X_{1}, \varepsilon_{1} \sigma_{1} \bar{\sigma}_{1}^{-1}\right)-\phi\left(X_{1}, \varepsilon_{1}\right)\right\} \times$

$E\left|\left\|X_{1}\right\|\right| \varepsilon_{1} \sigma_{1}|| \bar{\sigma}_{1}-\sigma_{1}\left|H\left(X_{1}\right) L\left(X_{1}\right)\right| \leq$ $\int_{0}^{1} \phi\left(\mathrm{X}_{1}, \varepsilon_{1} \sigma_{1}\left(\tau \bar{\sigma}_{1}^{-1}+(1-\tau) \sigma_{1}^{-1}\right)\right) \mathrm{d} \tau \leq$ $\left\{E\left|\left\|X_{1}\right\|\right| \varepsilon_{1} \sigma_{1}\left|H\left(X_{1}\right) L\left(X_{1}\right)\right|^{v /(v-2)}\right\}^{(v-2) / v}\left\{E\left|\vec{\sigma}_{1}^{2}-\sigma_{1}^{2}\right|^{v / 4}\right\}^{2 / v}=o(1)$

by Lemma 1. Now note that the left hand side of (b.13) is bounded by, 


$$
\left\{\min _{i} \hat{\sigma}_{i} \min _{i} \tilde{\sigma}_{i}\left(\min _{i} \tilde{\sigma}_{i}+\min _{i} \hat{\sigma}_{i}\right)\right\}^{-1}\left\{\sum_{i}\left|\hat{\sigma}_{i}^{2}-\tilde{\sigma}_{i}^{2}\right|^{2}\right\}^{1 / 2}\left\{n^{-1} \Sigma_{i} L\left(x_{i}\right)^{2}\right\}^{1 / 2}=o_{p}(1)
$$

by lemmas 5,6 and 7 . The left hand side of (b.14) is bounded by,

$$
\left\{\min _{i} \tilde{\sigma}_{i} \min _{i} \bar{\sigma}_{i}\right\}^{-1} n^{-1 / 2} \sum_{i} L\left(X_{i}\right)\left|\tilde{\sigma}_{i}-\bar{\sigma}_{i}\right|=o_{p}(1),
$$

by Lemmas 4 and 5 and Chevyshev's inequality, since,

$$
E\left|\mathrm{n}^{-1 / 2} \sum_{i} L\left(X_{i}\right)\right| \tilde{\sigma}_{i}-\bar{\sigma}_{i}||^{2} \leq C_{1}+C_{2}
$$

where, by Hölder's inequality,

$C_{1}=E\left\{L\left(X_{1}\right)^{2}\left|\tilde{\sigma}_{1}-\bar{\sigma}_{1}\right|^{2}\right\} \leq\left\{E\left|L\left(X_{1}\right)\right|^{2(v-2) / v}\right\}^{v /(v-2)}\left\{E\left|\tilde{\sigma}_{1}^{2}-\bar{\sigma}_{1}^{2}\right|^{v / 2}\right\}^{2 / v}=O\left(k^{-1 / 2}\right)$

and by Cauchy's and Hölder's inequalities,

$$
\begin{aligned}
C_{2}=E\left\{n^{-1} \sum \sum_{i \neq j} L\left(X_{i}\right)\left|\tilde{\sigma}_{i}-\bar{\sigma}_{i}\right| L\left(X_{j}\right)\left|\tilde{\sigma}_{j}-\bar{\sigma}_{j}\right|\right\} \leq E\left[n^{-1} \sum_{i \neq j} \sum_{i} L\left(X_{i}\right)^{2}\left|\tilde{\sigma}_{j}-\bar{\sigma}_{j}\right|^{2}\right] \\
\leq\left\{E\left|L\left(X_{1}\right)\right|^{2 v /(v-2)}\right\}^{(v-2) / v}\left\{n E\left|\tilde{\sigma}_{1}^{2}-\bar{\sigma}_{1}^{2}\right|^{v / 2}\right\}^{2 / v}=O\left[\left(n k^{-v / 4}\right)^{2 / v}\right]
\end{aligned}
$$

by Lemma 8 and $\mathrm{R} 4$. Now note that,

$\left\|\mathrm{n}^{-1 / 2} \sum_{\mathrm{i}} \phi\left(\mathrm{X}_{\mathrm{i}}, \varepsilon_{\mathrm{i}} \sigma_{\mathrm{i}} \hat{\sigma}_{\mathrm{i}}^{-1}\right)-\phi\left(\mathrm{X}_{\mathrm{i}}, \varepsilon_{\mathrm{i}} \sigma_{\mathrm{i}} \bar{\sigma}_{\mathrm{i}}^{-1}\right)\right\|=$

$\left\|n^{-1 / 2} \sum_{i} \varepsilon_{i} \sigma_{i}\left(\hat{\sigma}_{i}^{-1}-\bar{\sigma}_{i}^{-1}\right) \int_{0}^{1} \phi^{\prime}\left(X_{i}, \varepsilon_{i} \sigma_{i}\left[\hat{\sigma}_{i}^{-1} \tau+\bar{\sigma}_{i}^{-1}(1-\tau)\right]\right) d \tau\right\|$

$\leq K n^{-1 / 2} \sum_{i}\left\|X_{i}\right\| H\left(X_{i}\right)\left|\varepsilon_{i} \sigma_{i}\right|\left|\hat{\sigma}_{i}^{-1}-\tilde{\sigma}_{i}^{-1}\right|+K n^{-1 / 2} \sum_{i}\left\|X_{i}\right\| H\left(X_{i}\right)\left|\varepsilon_{i} \sigma_{i}\right|\left|\hat{\sigma}_{i}^{-1}-\tilde{\sigma}_{i}^{-1}\right|$

$=o_{p}(1)$,

using arguments in (b.13) and (b.14). Then by (b.9), $U\left(\Delta^{*}, \hat{\sigma}\right)=\mathrm{op}(1)$ and

$$
0=\left\|U_{n}\left[{ }^{1 / 2}\left(\check{\theta}_{n}(\hat{\sigma})-\theta^{o}\right), \hat{\sigma}\right]\right\| \leq 2\left\|U_{n}\left(\Delta^{*}, \sigma\right)\right\|=o_{p}(1),
$$

and given (b.9), $n^{1 / 2}\left(\check{\theta}_{n}(\hat{\sigma})-\theta^{\circ}\right)=O_{p}(1)$, since for each $\eta>0, \tau>0$ and $M$, there exists a Mı satisfying,

$$
\operatorname{Pr}\left\{\inf _{\|\Delta\| \leq M_{1}}\left\|U_{n}(\Delta, \hat{\sigma})\right\|>\eta\right\}>1-\tau,
$$

using same arguments as Juređkovà's (1977) proof of her Lemma 5.2. By (b.18),

$$
n^{1 / 2}\left(\check{\theta}_{n}(\hat{\sigma})-\theta^{0}\right)=V(\sigma)^{-1} U_{n}(0, \sigma)+o_{p}(1) \rightarrow_{d} N\left(0, \Phi_{1}\right) \text {, }
$$

by the clt.

Proof of Theorem 2. (b): Following arguments given in the proof to Theorem 2, it suffices to prove that,

$$
\sup _{\|\Delta\| \leq M}\left\|U_{n}(\Delta, \check{\sigma})-U_{n}(0, \check{\sigma})-\left\{U_{n}(\Delta, \sigma)-U_{n}(0, \sigma)\right\}\right\|=o_{p}(1),
$$


and

$$
\left\|U_{n}(\Delta, \check{\sigma})-U_{n}(0, \sigma)\right\|=o_{p}(1) .
$$

(b.19) and (b.20) follow in a similar manner as (b.2) and (b.10) but using the following Lemmas proved in Delgado (1989),

Lemma 12. $\operatorname{Pr}\left\{\min _{i} \dot{\sigma}_{i} \leq \delta\right\}=0$ all $\mathrm{n}$ and some $\delta>0$.

Lemma 13. - $\left\{\min \quad \check{\sigma}^{2}\right\}^{-1}=0(1)$.

Lemma 14. $E\left\{\left|\check{\sigma}_{1}^{2}-\dot{\sigma}_{1}^{2}\right|^{v / 2}\right\}=O\left(k^{-v / 4}\right)$.

Lemma 15.- $\max _{i}\left|\breve{\sigma}_{i}^{2}-\dot{\sigma}_{i}^{2}\right|=o_{p}\left(k^{-1 / 2}\right)$.

where $\dot{\sigma}_{i}^{2}=\sum_{j} E\left(Y_{j}^{2} \mid X_{j}\right) \omega_{i j}-\left\{\sum_{j} X_{j}^{\prime} \theta^{\circ} \omega_{i j}\right\}^{2}$.

Acknowledgements: This work was started at the London School of Economics as part of my doctoral dissertation. I thank Peter Robinson for encouraging me to investigate this problem. Financial support from the Economic and Social Research Council (ESRC) reference numbers: B00232156 and R000231441 and from a Indiana University Faculty Grant is gratefully acknowledged.

\section{References}

Carroll, R.J. (1982), "Adapting for heteroscedasticity in linear models", Annals of Statistics 10, 1244-1233.

Carroll, R.J. and D. Ruppert (1982), "Robust estimation in heteroscedastic linear models", Annals of Statistics 10,429-441.

Delgado, M.A. (1989), "An alternative asymptotically efficient semiparametric .generalised least squares estimator", Unpublished.

Hampel, F.R., E.M. Rouchetti, P.J. Rousseew and W.A. Stahel (1986), Robust Statistics, Wiley: New York.

Huber, P.J. (1973), "Robust regression: asymptotics, conjectures and Monte

Carlo", Annals of Statistics 1, 799-821.

Jureckova, J. (1977), "Asymptotic relations of M-estimates and R-estimates in linear regression model", Annals of Statistics 5,464-472.

Maronna, R.A. and V.J. Yohai, "Asymptotic behaviour of general M-estimates for regression and scale with random carriers", Zeitschrift für

Wahrscheinlichkeitstheorie und verwandte Gebiete 58, 7-20

Relles, D. (1968), Robust Regression by Modified Least Squares, Ph.D. Thesis, Yale University.

Robinson, P.M. (1987), "Asymptotically efficient estimation in the presence of heteroskedasticity of unknown form", Econometrica 55, 875-891.

Rose, R.L. (1978), Nonparametric Estimation of Weights in Least-Squares

Regression Analysis, Ph.D. Thesis, University of California at Davis.

Stone, C.J. (1977), "Consistent nonparametric regression", (with discussion) Annals of Statistics 5,595-645.

Yohai, V.J. and R.A. Maronna (1979), "Asymptotic behavior of M-estimators for the linear model", Annals of Statistics 7, 258-268. 
TABLE 1

CONTAMINATED ERRORS

MODEL $1(\gamma=0$.

\begin{tabular}{|c|c|c|c|c|c|c|c|c|c|}
\hline & \multicolumn{3}{|c|}{$\mathrm{N}=30$} & \multicolumn{3}{|c|}{$\mathrm{N}=100$} & \multicolumn{3}{|c|}{$N=500$} \\
\hline & BIAS & VAR & EFF & BIAS & VAR & EFF & BIAS & VAR & EFF \\
\hline$(\sigma)$ & $-5 E-5$ & .1028 & 1.0000 & -.0045 & .0317 & 1.0000 & $-6 E-4$ & .0062 & 1.0000 \\
\hline 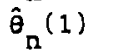 & $-5 E-5$ & .1028 & 1.0000 & -.0045 & .0317 & 1.0000 & $-6 E-4$ & .0062 & 1.0000 \\
\hline E & -.0027 & .1007 & 1.0204 & .0022 & 0282 & 1. 1235 & -.0016 & .0059 & 1.0528 \\
\hline$(\hat{\sigma})^{1}$ & -.0015 & .3541 & 2903 & -.0043 & .0569 & .5564 & $6 E-4$ & .0078 & .7965 \\
\hline$(\hat{\sigma})^{2}$ & .0031 & .3073 & .3348 & $-6 E-4$ & .0488 & .6483 & $-4 E-4$ & .0069 & .8961 \\
\hline$(\check{\sigma})^{1}$ & .0026 & .3703 & 2776 & -.0032 & .0585 & .5415 & $8 E-4$ & .0079 & .7908 \\
\hline$(\check{\sigma})^{2}$ & .0132 & .2881 & 3566 & -.0025 & .0477 & .6639 & $-3 E-4$ & .0069 & .9009 \\
\hline$(\sigma)$ & $-9 E-4$ & .0766 & 1.3416 & -.0025 & .0220 & 1.4392 & $-8 E-4$ & .0043 & 1.4327 \\
\hline$n^{(1)}$ & $-9 E-4$ & .0766 & 1. 3416 & -.0025 & .0220 & 1.4392 & $-8 E-4$ & .0043 & 1. 4327 \\
\hline$n(\hat{\sigma})^{1}$ & $2 E-4$ & .1488 & .6909 & -.006 & .030 & 1.0429 & $-7 E-4$ & .0050 & 1.2353 \\
\hline$n(\hat{\sigma})^{2}$ & $3 E-4$ & 1136 & .9053 & -.003 & .027 & 1. 1471 & -.0012 & .0049 & 1.2697 \\
\hline$\ddot{\theta}_{n}(\check{\sigma})^{1}$ & $6 E-4$ & .1370 & .7501 & -.004 & .029 & 1.0752 & $-7 E-4$ & .0050 & 1.2413 \\
\hline$\check{\theta}_{n}(\check{\sigma})^{2}$ & .0135 & .1085 & .9464 & -.001 & .027 & 1.177 & -.0011 & .0048 & $1.2757^{\circ}$ \\
\hline
\end{tabular}

MODEL $1(\gamma=-1$.

\begin{tabular}{|c|c|c|c|c|c|c|c|c|c|}
\hline & \multicolumn{3}{|c|}{$N=30$} & \multicolumn{3}{|c|}{$N=100$} & \multicolumn{3}{|c|}{$\mathrm{N}=500$} \\
\hline & BIAS & VAR & EFF & BIAS & VAR & EFF & BIAS & VAR & EFF \\
\hline$(\sigma)$ & $-2 E-5$ & .0146 & 1.0000 & $4 E-4$ & .0046 & 1.0000 & $-6 E-4$ & $88 \mathrm{E}-5$ & 1.0000 \\
\hline (1) & .0026 & .0339 & 4310 & -.0031 & .0096 & .4816 & $-7 \mathrm{E}-7$ & .0020 & .4384 \\
\hline & $-5 E-4$ & .0193 & .7594 & $-5 E-4$ & .0053 & .8824 & $-9 E-4$ & .0010 & .8064 \\
\hline$(\hat{\sigma})^{1}$ & $-5 E-4$ & .0529 & .2767 & .0014 & .0086 & .5391 & $-4 E-4$ & .0011 & .7808 \\
\hline$(\hat{\tilde{\sigma}})$ & .0017 & .0430 & .3405 & $-2 E-5$ & .0073 & .6342 & $-7 E-4$ & $99 E-4$ & .8942 \\
\hline$(\dot{\sigma})^{1}$ & .0044 & .0578 & .2533 & .0014 & .0091 & .5129 & $-2 E-4$ & .0011 & .7785 \\
\hline$n(\check{\sigma})^{2}$ & .0094 & .0430 & .3402 & .0010 & .0069 & .6669 & $-7 E-4$ & $97 E-4$ & .9075 \\
\hline$(\sigma)$ & -.0010 & .0109 & 1. 3354 & $-3 E-4$ & .0032 & 1.4432 & $-9 E-4$ & $62 E-5$ & 1. 4167 \\
\hline$n(1)$ & .0016 & .0257 & .5697 & -.0026 & .0072 & .6453 & $-9 E-4$ & .0014 & .6098 \\
\hline$n(\hat{\sigma})^{1}$ & $-3 E-4$ & .0217 & .6738 & $-6 E-4$ & .0046 & 1.0182 & -.0013 & $71 E-5$ & 1.2382 \\
\hline$n^{(\hat{\sigma})^{2}}$ & $5 E-4$ & .0176 & .8317 & $-3 E-4$ & .0042 & 1.1051 & -.0012 & $69 E-5$ & 1.2695 \\
\hline$\breve{\theta}_{n}(\check{\sigma})^{1}$ & .0044 & .0210 & .6959 & $3 E-4$ & .0045 & 1.0351 & -.0012 & $71 E-5$ & 1.2381 \\
\hline$\dot{\theta}_{n}(\check{\sigma})^{2}$ & .0087 & .0187 & .7822 & $4 E-4$ & .0043 & 1.0675 & -.0011 & $70 E-5$ & 1.2574 \\
\hline
\end{tabular}


TABLE 1 (Cont.)

MODEL $2(\delta=3$.

\begin{tabular}{|c|c|c|c|}
\hline & \multicolumn{3}{|c|}{$\mathrm{N}=30$} \\
\hline & BIAS & VAR & EFF \\
\hline$\hat{\theta}_{n}(\sigma)$ & -.0015 & 4.8231 & 1.0000 \\
\hline$\hat{\theta}_{n}(1)$ & -.0653 & 23. 390 & .2061 \\
\hline LADE & -.0455 & 9.0602 & .5322 \\
\hline$\hat{\theta}_{n}(\hat{\sigma})^{1}$ & -.0397 & 19. 105 & .2524 \\
\hline$\hat{\theta}_{n}(\hat{\sigma})^{2}$ & -.0320 & 16.698 & .2888 \\
\hline$\hat{\theta}_{n}^{11}(\dot{\sigma})^{1}$ & -.0519 & 21.121 & .2283 \\
\hline$\hat{\theta}_{n}^{1}(\check{\sigma})^{2}$ & -.0290 & 16.021 & .3010 \\
\hline$\ddot{\theta}_{n}(\sigma)$ & .0139 & 3.6326 & 1. 3276 \\
\hline$\check{\theta}_{n}(1)$ & -.0487 & 8.7229 & .5527 \\
\hline$\ddot{\theta}_{n}(\hat{\sigma})^{1}$ & $-2 E-4$ & 7.7019 & .6262 \\
\hline$\ddot{\theta}_{n}(\hat{\sigma})^{2}$ & -.0290 & 6.9172 & .6971 \\
\hline$\check{\theta}_{n}(\check{\sigma})^{1}$ & .0117 & 7.3163 & .6592 \\
\hline$\ddot{\theta}_{n}(\check{\sigma})^{2}$ & -.0063 & 6.5064 & .7413 \\
\hline
\end{tabular}
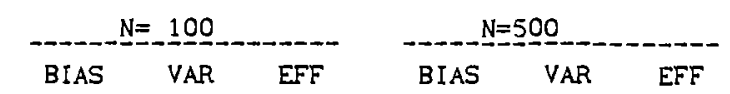

$\begin{array}{lllllll}-.0361 & 1.3672 & 1.0000 & .0197 & .2781 & 1.0000\end{array}$

$\begin{array}{lllllll}-.0348 & 6.9974 & .1975 & -.0172 & 1.3980 & .1992\end{array}$

$\begin{array}{lllllll}.0054 & 2.4612 & .5560 & -.0051 & .4824 & .5772\end{array}$

$\begin{array}{llllll}-.0245 & 2.6323 & .5197 & .0322 & .3846 & .7222\end{array}$

$\begin{array}{lllllll}-.0268 & 2.2223 & .6156 & .0226 & .3381 & .8226\end{array}$

$\begin{array}{llllll}-.0249 & 2.7676 & .4943 & .0304 & .3895 & .7132\end{array}$

$\begin{array}{lllllll}-.0276 & 2.2027 & .6211 & .0220 & .3375 & .8238\end{array}$

$\begin{array}{lllllll}-.0236 & 1.0115 & 1.3522 & .0219 & .1975 & 1.4067\end{array}$

$\begin{array}{llllll}.0043 & 2.4801 & .5517 & -5 E-4 & .4954 & .5622\end{array}$

$\begin{array}{lllllll}-.0132 & 1.4622 & .9357 & .0354 & .2500 & 1.1084\end{array}$

$\begin{array}{lllllll}-.0159 & 1.3926 & .9825 & .0250 & .2406 & 1.1543\end{array}$

$\begin{array}{lllllll}-.0082 & 1.4371 & .9522 & .0344 & .2487 & 1.1144\end{array}$

$\begin{array}{lllllll}-.0163 & 1.3660 & 1.0016 & .0245 & .2401 & 1.1573\end{array}$

MODEL $2(\delta=5$.

\begin{tabular}{|c|c|c|c|}
\hline & \multicolumn{3}{|c|}{$N=30$} \\
\hline & BIAS & VAR & EFF \\
\hline$\hat{\theta}_{n}(\sigma)$ & .0194 & 39.714 & 1.0000 \\
\hline$\hat{\theta}_{n}(1)$ & -.5159 & 1461.4 & .0271 \\
\hline LADE & -.2619 & 258.41 & .1536 \\
\hline$\hat{\theta}_{n}(\hat{\sigma})^{1}$ & -.1122 & 206.76 & .1920 \\
\hline$\hat{\theta}_{n}^{n}(\hat{\sigma})^{2}$ & -.1928 & 231.36 & .1716 \\
\hline$\hat{\theta}_{n}(\check{\sigma})^{1}$ & -.1644 & 216.73 & .1832 \\
\hline$\hat{\theta}_{n}(\dot{\sigma})^{2}$ & -.2036 & 211.56 & .1876 \\
\hline$\check{\theta}_{n}(\sigma)$ & .0720 & 30.293 & 1. 3107 \\
\hline$\dot{\theta}_{n}(1)$ & -.2749 & 256.30 & .1549 \\
\hline$\check{\theta}_{n}(\hat{\sigma})^{1}$ & .0353 & 82.535 & .4812 \\
\hline$\check{\theta}_{n}(\hat{\sigma})^{2}$ & -.1151 & 99.400 & .3995 \\
\hline$\ddot{\theta}_{n}\left(\check{\sigma}^{1}\right.$ & .0584 & 75.235 & .5276 \\
\hline$\ddot{\theta}_{n}(\check{\sigma})^{2}$ & -.0652 & 89.291 & .4447 \\
\hline
\end{tabular}
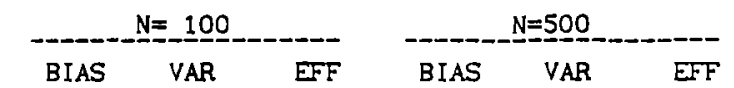

$\begin{array}{llllll}-.1011 & 9.8887 & 1.0000 & .0592 & 1.8264 & 1.0000\end{array}$

$\begin{array}{llllll}-.2760 & 442.68 & .0223 & .1996 & 88.534 & .0207\end{array}$

$\begin{array}{llllll}.0603 & 56.269 & .1759 & .0139 & 9.4482 & .1936\end{array}$

$\begin{array}{lllllll}-.0536 & 21.595 & .4583 & .0848 & 2.6485 & .6890\end{array}$

$\begin{array}{lllllll}-.1033 & 19.826 & .4990 & .0598 & 2.3742 & .7695\end{array}$

$\begin{array}{lllllll}-.0530 & 21.978 & .4503 & .0783 & 2.6674 & .6844\end{array}$

$\begin{array}{lllllll}-.1026 & 19.534 & .5065 & .0569 & 2.3597 & .7744\end{array}$

$\begin{array}{lllllll}-.0727 & 7.3305 & 1.3494 & .0658 & 1.2893 & 1.4144\end{array}$

$\begin{array}{llllll}.0464 & 57.008 & .1736 & .0047 & 9.9333 & .1842\end{array}$

$\begin{array}{lllllll}-.0227 & 11.716 & .8448 & .1010 & 1.7162 & 1.0599\end{array}$

$\begin{array}{lllllll}-.0634 & 12.559 & .7879 & .0737 & 1.6991 & 1.0735\end{array}$

$\begin{array}{lllllll}-.0182 & 11.026 & .8978 & .0982 & 1.6752 & 1.0861\end{array}$

$\begin{array}{lllllll}-.0620 & 12.217 & .8100 & .0719 & 1.6848 & 1.0827\end{array}$ 
TABLE 1 (Cont.)

MODEL $1 \quad(\gamma=-2$. $)$

\begin{tabular}{lccc} 
& & \multicolumn{2}{c}{ N=330 } \\
& BIAS & VAR & EFF \\
$\hat{\theta}_{n}(\sigma)$ & $-3 E-6$ & .0016 & 1.0000 \\
$\hat{\theta}_{n}(1)$ & .0028 & .0227 & .0701 \\
LADE & $3 E-4$ & .0047 & .3359 \\
$\hat{\theta}_{n}(\hat{\sigma})^{1}$ & $-3 E-4$ & .0065 & .2449 \\
$\hat{\theta}_{n}(\hat{\sigma})^{2}$ & $6 E-4$ & .0056 & .2826 \\
$\hat{\theta}_{n}(\check{\sigma})^{1}$ & .0041 & .0172 & .0931 \\
$\hat{\theta}_{n}(\check{\sigma})^{2}$ & .0107 & .0192 & .0829 \\
$\check{\theta}_{n}(\sigma)$ & $-3 E-4$ & .0012 & 1.3003 \\
$\check{\theta}_{n}(1)$ & .0021 & .0174 & .0920 \\
$\check{\theta}_{n}(\hat{\sigma})^{1}$ & $-3 E-4$ & .0027 & .599 \\
$\check{\theta}_{n}(\hat{\sigma})^{2}$ & $5 E-5$ & .0025 & .6466 \\
$\dot{\theta}_{n}(\check{\sigma})^{1}$ & .0028 & .0051 & .3122 \\
$\check{\theta}_{n}(\check{\sigma})^{2}$ & .0084 & .0068 & .2326
\end{tabular}

\begin{tabular}{|c|c|c|c|c|c|}
\hline \multicolumn{3}{|c|}{$N=100$} & \multicolumn{3}{|c|}{$N=500$} \\
\hline BIAS & VAR & EFF & BIAS & VAR & $\mathrm{EFF}$ \\
\hline $6 E-4$ & $46 E-5$ & 1.0000 & $-1 E-4$ & $95 E-6$ & 1.0000 \\
\hline-.0028 & .0063 & .0725 & $-4 E-4$ & .0013 & .0697 \\
\hline$-5 E-5$ & .0012 & .3791 & $-5 E-4$ & $23 E-5$ & .4103 \\
\hline .0010 & $98 \mathrm{E}-5$ & 4681 & $-3 E-5$ & $12 E-5$ & .7513 \\
\hline $1 E-4$ & $78 E-5$ & 5868 & $-1 E-5$ & $11 E-5$ & .8546 \\
\hline $9 E-4$ & .0014 & 3355 & $-3 E-4$ & $13 E-5$ & .7229 \\
\hline $6 \mathrm{E}-4$ & .0016 & .2841 & $-4 \mathrm{E}-4$ & $15 E-5$ & 6048 \\
\hline $3 E-4$ & $32 E-5$ & 1. 3991 & $-2 E-4$ & $68 \mathrm{E}-6$ & 1.4010 \\
\hline-.0022 & .0047 & 0974 & $-6 E-4$ & $97 \mathrm{E}-5$ & .0977 \\
\hline $4 E-4$ & $50 E-5$ & .9189 & $-3 E-4$ & $79 E-6$ & 1. 1982 \\
\hline $2 E-4$ & $46 E-5$ & 1.0006 & $-3 E-4$ & $77 \mathrm{E}-6$ & 1.2366 \\
\hline $4 E-4$ & $74 E-4$ & .6718 & $-5 E-4$ & $87 \mathrm{E}-6$ & 1.0921 \\
\hline $5 E-4$ & .0010 & .4450 & $-6 E-4$ & $11 E-5$ & 53 \\
\hline
\end{tabular}

MODEI $2(\delta=1$.

\begin{tabular}{|c|c|c|c|c|c|c|c|c|c|}
\hline & \multicolumn{3}{|c|}{$N=30$} & \multicolumn{3}{|c|}{$N=100$} & \multicolumn{3}{|c|}{$\mathrm{N}=500$} \\
\hline & BIAS & VAR & EFF & BIAS & VAR & $\mathrm{EFF}$ & BIAS & VAR & EFF \\
\hline . & $-4 E-5$ & .3627 & 1.0000 & .0067 & .1061 & 1.0000 & .0015 & .0222 & .0000 \\
\hline & -.0057 & .4716 & .7691 & .0047 & .1399 & 7588 & $3 E-4$ & .0283 & .7836 \\
\hline$n$ & -.0079 & .3865 & .9383 & .0024 & .1123 & .9455 & -.0022 & .0227 & .9779 \\
\hline & -.0053 & 1.2806 & .2832 & -.0056 & .2016 & .5269 & .0042 & .0286 & .7750 \\
\hline 2 & .0018 & 1. 1202 & .3237 & -.0037 & .1692 & .6278 & .0019 & .0254 & .8714 \\
\hline$(\check{\sigma})^{1}$ & -.0061 & 1.3736 & 2640 & -.0048 & .2065 & .5143 & .0042 & .0289 & .7661 \\
\hline$)^{2}$ & .0089 & 1.0821 & .3352 & -.0025 & .1670 & .6358 & .0019 & .0254 & .8738 \\
\hline & $5 E-4$ & .2715 & 1.3357 & -.0042 & .0778 & 1.3637 & -.0015 & .0155 & 1.4262 \\
\hline & -.0070 & .3113 & 1.1649 & -.0010 & .0914 & 1.1616 & -.0013 & .0189 & 1.1709 \\
\hline$\hat{1}, 1$ & $-4 E-4$ & .5389 & .6731 & -.0040 & .1101 & .9640 & .0031 & .0186 & 1.1962 \\
\hline & -.0036 & .4175 & .8688 & -.0027 & .0979 & 1.0842 & -.0013 & .0179 & 1.2353 \\
\hline$(\bar{\sigma})^{1}$ & .0064 & .5006 & .7244 & -.0037 & .1072 & .9902 & .0030 & .0185 & 1.1962 \\
\hline$(\check{\sigma})^{2}$ & .0087 & .3956 & .9166 & -.0018 & .0960 & 1.1063 & -.0013 & .0179 & 1.2353 \\
\hline
\end{tabular}


TABLE 2

NORMAL ERRORS

MODEI I $(\gamma=-2$.

$\begin{array}{lccc} & \text { BIAS } & \text { VAR } & \text { EFF } \\ \hat{\theta}_{n}(\sigma) & -2 E-4 & .0016 & 1.0000 \\ \hat{\theta}_{n}(1) & .0028 & .0448 & .0707 \\ \text { LADE } & 1 E-4 & .0072 & .2232 \\ \hat{\theta}_{n}(\hat{\sigma})^{1} & 1 E-4 & .0049 & .3277 \\ \hat{\theta}_{n}(\hat{\sigma})^{2} & 8 E-4 & .0039 & .4121 \\ \hat{\theta}_{n}(\check{\sigma})^{1} & .0048 & .0118 & .1369 \\ \hat{\theta}_{n}(\check{\sigma})^{2} & .0117 & .0122 & .1311 \\ \dot{\theta}_{n}(\sigma) & -3 E-4 & .0017 & .9637 \\ \dot{\theta}_{n}(1) & .0025 & .0217 & .0746 \\ \dot{\theta}_{n}(\hat{\sigma})^{1} & 1 E-4 & .0031 & .5201 \\ \dot{\theta}_{n}(\hat{\sigma})^{2} & 9 E-5 & .0029 & .5729 \\ \check{\theta}_{n}(\check{\sigma})^{1} & .0034 & .0060 & .2692 \\ \hat{\theta}_{n}(\check{\sigma})^{2} & .0084 & .0079 & .2039\end{array}$

\begin{tabular}{rccccc}
\hline BIAS & VAR & EFF & BIAS & VAR & EFF \\
$-2 E-4$ & $46 E-5$ & 1.0000 & $-2 E-4$ & $92 E-6$ & 1.0000 \\
$-7 E-4$ & .0069 & .0675 & $-7 E-4$ & .0013 & .0681 \\
$-7 E-5$ & .0019 & .2458 & $-6 E-4$ & $35 E-5$ & .2581 \\
$-7 E-5$ & $64 E-5$ & .7198 & $-1 E-4$ & $10 E-5$ & .9057 \\
$6 E-4$ & $56 E-5$ & .8225 & $-3 E-4$ & $97 E-6$ & .9500 \\
$5 E-4$ & .0010 & .4638 & $-4 E-4$ & $11 E-5$ & .8439 \\
$8 E-4$ & .0013 & .3543 & $-6 E-4$ & $14 E-5$ & .6584 \\
$1 E-4$ & $48 E-5$ & .9653 & $-3 E-4$ & $98 E-6$ & .9443 \\
$-6 E-4$ & .0064 & .0725 & $-8 E-4$ & .0013 & .0733 \\
$-1 E-4$ & $58 E-5$ & .8042 & $-3 E-4$ & $10 E-5$ & .9087 \\
$2 E-5$ & $54 E-5$ & .8647 & $-3 E-4$ & $97 E-6$ & .9487 \\
$2 E-4$ & $85 E-5$ & .5465 & $-5 E-4$ & $11 E-5$ & .8598 \\
$7 E-4$ & .0012 & .3959 & $-6 E-4$ & $14 E-5$ & .6583
\end{tabular}

MODEL $2(\delta=5$.

\begin{tabular}{|c|c|c|c|c|c|c|c|c|c|}
\hline \multirow[b]{2}{*}{ " } & \multicolumn{3}{|c|}{$N=30$} & \multicolumn{3}{|c|}{$N=100$} & \multicolumn{3}{|c|}{$N=500$} \\
\hline & BIAS & VAR & EFF & BIAS & VAR & EFF & BIAS & VAR & EFF \\
\hline$\hat{\theta}_{n}(\sigma)$ & .0176 & 39.134 & 0000 & 2 & 03 & 10000 & 7 & 0 & Dooo \\
\hline (1) & -.5878 & 1490.7 & .0262 & 2371 & 441.68 & .0223 & 2241 & 7.482 & .0201 \\
\hline$D E$ & -.3699 & 394.42 & .0992 & .0495 & 86.638 & .1137 & .0062 & 14.855 & .1183 \\
\hline$(\hat{\sigma})^{1}$ & .0112 & 160.79 & .2434 & -.0311 & 15.677 & .6282 & .0754 & 2.0997 & .8350 \\
\hline$(\hat{\sigma})^{2}$ & -.0714 & 162.41 & .2409 & -.1191 & 15.872 & .6200 & .0624 & 2.0867 & .8409 \\
\hline$(\dot{\sigma})^{1}$ & -.0039 & 158.70 & .2466 & -.0149 & 15.011 & .6561 & .0688 & 2.0572 & .8526 \\
\hline$a(\dot{\sigma})^{2}$ & -.0192 & 142.78 & .2741 & -.1137 & 31 & .6377 & .0586 & 2.0709 & .8475 \\
\hline & .0546 & 40.576 & .9644 & -.0918 & 10.283 & 9570 & .0711 & 92 & .9481 \\
\hline & -.3630 & 391.76 & .0999 & -.0363 & 09 & .1 & $7 E-4$ & 15.251 & .115 \\
\hline$a^{(\hat{\sigma})^{2}}$ & .0569 & 99.315 & .3940 & -.0354 & 13.701 & .7188 & .0859 & 2.0444 & .8568 \\
\hline$a^{(\hat{\sigma})^{2}}$ & -.0367 & 117.45 & .3331 & -.1047 & 14.854 & .6626 & .0668 & 2.0589 & .8520 \\
\hline$(\dot{\sigma})^{1}$ & .0663 & 91.335 & .4284 & -.0169 & 12.966 & .7596 & .0795 & 2.0009 & .8758 \\
\hline$(\check{\sigma})^{2}$ & .0094 & 104.85 & .3732 & -.0990 & 14.465 & .6804 & .0636 & 2. 0433 & .8587 \\
\hline
\end{tabular}

